\title{
The Equivalence Problem for Deterministic MSO Tree Transducers is Decidable
}

\author{
Joost Engelfriet $^{1}$ and Sebastian Maneth ${ }^{2}$ \\ 1 LIACS, Leiden University, The Netherlands engelfri@liacs.nl \\ 2 Faculté I \& C, EPFL, Switzerland sebastian.maneth@epfl.ch
}

\begin{abstract}
It is decidable for deterministic MSO definable graph-to-string or graphto-tree transducers whether they are equivalent on a context-free set of graphs.
\end{abstract}

It is well known that the equivalence problem for nondeterministic (one-way) finite state transducers is undecidable, even when they cannot read or write the empty string [Gri68]. In contrast, equivalence is decidable for deterministic finite state transducers, even for two-way transducers [Gur82]. The question arises whether these results can be generalized from strings to transducers working on more complex structures like, e.g., trees or graphs. There is no accepted notion of finite state transducer working on graphs; instead, it is believed that transductions expressed in monadic second-order logic (MSO) are the natural counterpart of finite state transductions on graphs. The idea is to define an output graph by interpreting fixed MSO formulas on a given input graph. In fact, if the input and output graphs of such an MSO graph transducer are strings, then the resulting transductions (in the deterministic case) are precisely the deterministic two-way finite state transductions [EH01]. Hence, by the above, equivalence is decidable for deterministic MSO string transducers. A nondeterministic MSO graph transducer can easily simulate a nondeterministic finite state transducer that cannot read the empty string; hence, equivalence is undecidable. Actually, even for deterministic MSO graph transducers equivalence is undecidable. This is due to the fact that MSO is undecidable for graphs (Propositions 5.21 and 5.2.2 of [Cou97]). The question remains whether deterministic MSO tree transducers have a decidable equivalence problem. Recently, these transducers have been characterized by certain attribute grammars [BE00] and macro tree transducers [EM99]. However, for both models it is unknown whether equivalence is decidable. Here we give an affirmative answer: equivalence of deterministic MSO tree transducers is decidable. This result has several applications; for instance, it implies that XML queries of linear size increase have decidable equivalence, by the results of [MSV03], [EM03a], [EM03b], and [Man03]. Our proof generalizes the one of [Gur82] (see also [Iba82]): it is based on the fact that certain sets are semilinear. The reader is assumed to be familiar with MSO on graphs and with MSO graph transducers, see, e.g., [Cou97,Cou94].

Convention: All lemmas stated in this paper are effective.

A graph alphabet is a pair $(\Sigma, \Gamma)$ of alphabets of node and edge labels, respectively. A graph over $(\Sigma, \Gamma)$ is a tuple $(V, E, \lambda)$ where $V$ is the finite set of nodes, $E \subseteq V \times$ $\Gamma \times V$ is the set of edges, and $\lambda: V \rightarrow \Sigma$ is the node labeling function. The set of all graphs over $(\Sigma, \Gamma)$ is denoted $\operatorname{GR}(\Sigma, \Gamma)$. The language $\operatorname{MSO}(\Sigma, \Gamma)$ of monadic 
second-order (MSO) formulas over $(\Sigma, \Gamma)$ uses node variables $x, y, \ldots$ and node-set variables $X, Y, \ldots$; both can be quantified with $\exists$ and $\forall$. It has atomic formulas $\operatorname{lab}_{\sigma}(x)$ for $\sigma \in \Sigma$, denoting that $x$ is labeled $\sigma$, $\operatorname{edg}_{\gamma}(x, y)$ for $\gamma \in \Gamma$, denoting that there is a $\gamma$-labeled edge from $x$ to $y$, and $x \in X$ denoting that $x$ is in $X$. For $g \in \operatorname{GR}(\Sigma, \Gamma)$ and a closed formula $\psi$ in $\operatorname{MSO}(\Sigma, \Gamma)$ we write $g \models \psi$ if $g$ satisfies $\psi$; similarly, if $\psi$ has free variables $x$ or $x, y$ and $u, v$ are nodes of $g$, then we write $(g, u) \models \psi$ or $(g, u, v) \models \psi$ if $g$ satisfies $\psi$ with $x=u$ or with $x=u, y=v$, respectively.

Let $\left(\Sigma_{1}, \Gamma_{1}\right),\left(\Sigma_{2}, \Gamma_{2}\right)$ be graph alphabets. A deterministic MSO graph transducer $M\left(\right.$ from $\left(\Sigma_{1}, \Gamma_{1}\right)$ to $\left.\left(\Sigma_{2}, \Gamma_{2}\right)\right)$ is a tuple $\left(C, \varphi_{\mathrm{dom}}, \Psi, X\right)$ where $C$ is a finite set of copy names, $\varphi_{\text {dom }} \in \operatorname{MSO}\left(\Sigma_{1}, \Gamma_{1}\right)$ is the closed domain formula, $\Psi=\left\{\psi_{c, \sigma}(x)\right\}_{c \in C, \sigma \in \Sigma_{2}}$ is a family of node formulas, i.e., MSO formulas $\psi_{c, \sigma}(x)$ over $\left(\Sigma_{1}, \Gamma_{1}\right)$ with one free variable $x$, and $X=\left\{\chi_{c, c^{\prime}, \gamma}(x, y)\right\}_{c, c^{\prime} \in C, \gamma \in \Gamma_{2}}$ is a family of edge formulas, i.e., MSO formulas $\chi_{c, c^{\prime}, \gamma}(x, y)$ over $\left(\Sigma_{1}, \Gamma_{1}\right)$ with two free variables $x, y$.

Given $g \in \operatorname{GR}\left(\Sigma_{1}, \Gamma_{1}\right)$, the graph $h=\tau_{M}(g) \in \operatorname{GR}\left(\Sigma_{2}, \Gamma_{2}\right)$ is defined if $g \models$ $\varphi_{\text {dom }}$, and then $V_{h}=\left\{(c, u) \mid c \in C, u \in V_{g}\right.$, there is exactly one $\sigma \in \Sigma_{2}$ such that $\left.(g, u) \models \psi_{c, \sigma}(x)\right\}, E_{h}=\left\{\left((c, u), \gamma,\left(c^{\prime}, u^{\prime}\right)\right) \mid(c, u),\left(c^{\prime}, u^{\prime}\right) \in V_{h}, \gamma \in \Gamma_{2}\right.$, and $\left.\left(g, u, u^{\prime}\right) \models \chi_{c, c^{\prime}, \gamma}(x, y)\right\}$, and $\lambda_{h}=\left\{((c, u), \sigma) \mid(c, u) \in V_{h}, \sigma \in \Sigma_{2}\right.$, and $\left.(g, u) \models \psi_{c, \sigma}(x)\right\}$. Hence, $\tau_{M}$ is a partial function from $\operatorname{GR}\left(\Sigma_{1}, \Gamma_{1}\right)$ to $\operatorname{GR}\left(\Sigma_{2}, \Gamma_{2}\right)$ with $\operatorname{dom}\left(\tau_{M}\right)=\left\{g \in \operatorname{GR}\left(\Sigma_{1}, \Gamma_{1}\right) \mid g \models \varphi_{\text {dom }}\right\}$.

A (nondeterministic) MSO graph transducer is obtained from a deterministic one by allowing all formulas to use fixed free node-set variables $Y_{1}, Y_{2}, \ldots$, called parameters. For each valuation of the parameters (by sets of nodes of the input graph) that satisfies the domain formula, the other formulas define the output graph as before. Hence each such valuation may lead to a different output graph for the given input graph. Thus, $\tau_{M} \subseteq \operatorname{GR}\left(\Sigma_{1}, \Gamma_{1}\right) \times \operatorname{GR}\left(\Sigma_{2}, \Gamma_{2}\right)$.

The following lemma contains a basic fact about MSO definable graph transductions; see, e.g., Proposition 3.2 in [Cou94].

Lemma 1. The (deterministic) MSO graph transductions are closed under composition.

Notation. Let $M_{1} ; M_{2}$ denote a transducer $M$ for which $\tau_{M}=\tau_{M_{2}} \circ \tau_{M_{1}}$; note that $M$ is deterministic, if $M_{1}$ and $M_{2}$ are. By Lemma $1, M_{1} ; M_{2}$ effectively exists.

In the sequel we often identify a transducer $M$ with its transduction $\tau_{M}$, and simply write, e.g., $M(g)$ in place of $\tau_{M}(g)$.

Let $M$ be an MSO graph transducer and let $X, Y$ be sets of graphs. Then $M$ is called an MSO $X$-to- $Y$ transducer, if $\operatorname{dom}(M) \subseteq X$ and range $(M) \subseteq Y$, and it is an MSO $X$ transducer if additionally $Y=X$.

A discrete graph (dgraph, for short) is a graph without edges. Let $g$ be a dgraph over $(\Sigma, \varnothing)$ with $\Sigma=\left\{\sigma_{1}, \ldots, \sigma_{k}\right\}$. Define $\operatorname{Par}(g)$ as the vector $\left(n_{1}, \ldots, n_{k}\right)$ in $\mathbb{N}^{k}$ such that, for $1 \leq i \leq k, n_{i}$ is the number of $\sigma_{i}$-labeled nodes in $g$. Similarly, for a string $w \in \Sigma^{*}, \operatorname{Par}(w)$ is the vector in $\mathbb{N}^{k}$ such that the $i$-th component is the number of $\sigma_{i}$ 's in $w$. We denote by $\operatorname{dgr}(w)$ the (unique) dgraph $g$ such that $\operatorname{Par}(g)=\operatorname{Par}(w)$. For a set $S$ of dgraphs or strings, $\operatorname{Par}(S)$ is the set of all $\operatorname{Par}(g)$ for $g \in S$. A set $P \subseteq \mathbb{N}^{k}$ is semilinear if there exists a regular language $R$ such that $P=\operatorname{Par}(R)$. The set $S$ is Parikh if $\operatorname{Par}(S)$ is semilinear. Note that since $\operatorname{Par}(R)=\emptyset$ iff $R=\emptyset$, emptiness of semilinear sets is decidable. 
A set of graphs is $N R$ if it is generated by a context-free node replacement graph grammar, see, e.g., [Eng97,Cou94]; it is also called C-edNCE or VR.

Lemma 2. (Theorem 7.1 of [Cou94]) The images of NR sets of graphs under MSO graph-to-dgraph transductions are Parikh.

In fact, the class of NR sets of graphs is closed under MSO graph transductions (see Theorem 4.2(3) of [Cou94], or Section 5 of [Eng97]) and NR sets of graphs are Parikh (see Proposition 4.11 of [Eng97]).

A useful property of semilinear sets is their (effective) closure under intersection. It implies the following lemma.

Lemma 3. It is decidable for a semilinear set $S \subseteq \mathbb{N}^{2}$ whether there exists an $n \in \mathbb{N}$ such that $(n, n) \in S$.

Proof. Let $P=\{(n, n) \mid n \in \mathbb{N}\}=\operatorname{Par}\left((a b)^{*}\right)$. The lemma holds because $S \cap P$ is semilinear [GS64] and semilinear sets have a decidable emptiness problem.

We identify the string $w=a_{1} a_{2} \cdots a_{n}$ with the graph that has \#-labeled nodes $v_{1}, \ldots, v_{n+1}$ and, for $1 \leq i \leq n$, an $a_{i}$-labeled edge from $v_{i}$ to $v_{i+1}$. For $1 \leq i \leq n$, we denote by $w / i$ the $i$-th letter $a_{i}$ of $w$.

Lemma 4. Let $\Delta$ be an alphabet and $a \in \Delta$. There exists an MSO string-to-dgraph transducer $N_{\Delta}^{a}$ such that for every $w \in \Delta^{*}$,

$$
N_{\Delta}^{a}(w)=\left\{\operatorname{dgr}\left(a^{n}\right) \mid w / n=a\right\} .
$$

Proof. The transducer $N_{\Delta}^{a}$ uses one parameter $Y_{1}$ to nondeterministically choose a node $v$ that has an outgoing $a$-labeled edge. It copies $v$ and all input nodes to the left of $v$, and labels them $a$. There are no edge formulas because dgraphs have no edges. Define $N_{\Delta}^{a}=\left(\{1\}, \varphi_{\text {dom }}\left(Y_{1}\right), \psi_{1, a}\left(x, Y_{1}\right), \varnothing\right)$ with

$$
\begin{aligned}
& \varphi_{\text {dom }}\left(Y_{1}\right) \equiv \operatorname{singleton}\left(Y_{1}\right) \wedge(\exists x)(\exists y)\left(\operatorname{edg}_{a}(x, y) \wedge x \in Y_{1}\right) \\
& \psi_{1, a}\left(x, Y_{1}\right) \equiv(\exists y)\left(x \preceq y \wedge y \in Y_{1}\right)
\end{aligned}
$$

where singleton $\left(Y_{1}\right)$ expresses that $Y_{1}$ is a singleton, and $x \preceq y$ that there is a path from $x$ to $y$.

We denote the disjoint union of graphs $h_{1}$ and $h_{2}$ by $h_{1} \uplus h_{2}$.

Lemma 5. Let $M_{1}, M_{2}$ be MSO graph transducers. There exists an MSO graph transducer $M$, denoted $M_{1} \uplus M_{2}$, such that for every graph $g$,

$$
M(g)=\left\{h_{1} \uplus h_{2} \mid h_{1} \in M_{1}(g), h_{2} \in M_{2}(g)\right\} .
$$

Proof. Let $M_{1}=\left(C_{1}, \varphi_{1}, \Psi_{1}, X_{1}\right)$ and $M_{2}=\left(C_{2}, \varphi_{2}, \Psi_{2}, X_{2}\right)$. We may assume w.l.o.g. that $C_{1}$ is disjoint from $C_{2}$ and that the parameters of $M_{1}$ are disjoint from those of $M_{2}$. Then $M=\left(C_{1} \cup C_{2}, \varphi_{1} \wedge \varphi_{2}, \Psi_{1} \cup \Psi_{2}, X_{1} \cup X_{2} \cup X\right)$ realizes the desired transduction, where all edge formulas in $X$ are set to false. 
Lemma 6. Let $M_{1}, M_{2}$ be MSO graph-to-string transducers and let $a, b$ be distinct symbols. There exists an MSO graph-to-dgraph transducer $M^{a, b}$ such that for every graph $g$,

$$
M^{a, b}(g)=\left\{\operatorname{dgr}\left(a^{m} b^{n}\right) \mid \exists h_{1} \in M_{1}(g), h_{2} \in M_{2}(g): h_{1} / m=a \text { and } h_{2} / n=b\right\} .
$$

Proof. Let $M_{i}$ be from $\left(\Sigma_{i}, \Gamma_{i}\right)$ to $\left(\{\#\}, \Delta_{i}\right)$ for $i \in\{1,2\}$. If $a \notin \Delta_{1}$ or $b \notin \Delta_{2}$ then let $M^{a, b}=(\varnothing$, false, $\varnothing, \varnothing)$. Otherwise define $M^{a, b}=\left(M_{1} ; N_{\Delta_{1}}^{a}\right) \uplus\left(M_{2} ; N_{\Delta_{2}}^{b}\right)$ according to Lemmas 1,4 , and 5 .

Let $\Sigma$ be a ranked alphabet, i.e., an alphabet $\Sigma$ together with a mapping $\operatorname{rank}_{\Sigma}$ : $\Sigma \rightarrow \mathbb{N}$. Let $m$ be the maximal rank of symbols in $\Sigma$. A tree (over $\Sigma$ ) is an acyclic, connected graph in $\operatorname{GR}(\Sigma,\{1, \ldots, m\})$, with exactly one node that has no incoming edges (the root), and, for $\sigma \in \Sigma$, every $\sigma$-labeled node has exactly $\operatorname{rank}_{\Sigma}(\sigma)$ outgoing edges, labeled $1,2, \ldots, \operatorname{rank}_{\Sigma}(\sigma)$, respectively.

For a relation $R \subseteq A \times B$ and a set $D \subseteq A$, denote by $\left.R\right|_{D}$ the restriction of $R$ to $D$, i.e., $\left.R\right|_{D}=\{(a, b) \in R \mid a \in D\}$.

Theorem 7. It is decidable for deterministic MSO graph-to-string or graph-to-tree transducers $M_{1}, M_{2}$ and an NR set $D$ of graphs whether $\left.\tau_{M_{1}}\right|_{D}=\left.\tau_{M_{2}}\right|_{D}$.

Proof. We start with the graph-to-string case. For $i \in\{1,2\}$ let $D_{i}=\operatorname{dom}\left(M_{i}\right) \cap D$. We first show that it is decidable whether $D_{1}=D_{2}$. Clearly, $D_{1}=D_{2}$ if and only if $\operatorname{Par}(E(D))=\emptyset$, where $E$ is the deterministic MSO graph-to-dgraph transducer that removes the edges of all graphs in the symmetric difference of $\operatorname{dom}\left(M_{1}\right)$ and $\operatorname{dom}\left(M_{2}\right)$ : $E=\left(\{1\}, \neg\left(\varphi_{1} \leftrightarrow \varphi_{2}\right),\left\{\psi_{1, \sigma}(x)\right\}_{\sigma \in \Sigma}, \emptyset\right\}$ where $\varphi_{i}$ is the domain formula of $M_{i}$ for $i \in\{1,2\}, \Sigma$ is the node alphabet of $D$, and $\psi_{1, \sigma}(x)=\operatorname{lab}_{\sigma}(x)$ for $\sigma \in \Sigma$. By Lemma 2, $\operatorname{Par}(E(D))$ is effectively semilinear, and hence its emptiness can be decided. If $D_{1} \neq D_{2}$ then we are finished and know that $\left.\tau_{M_{1}}\right|_{D} \neq\left.\tau_{M_{2}}\right|_{D}$. Assume now that $D_{1}=D_{2}$.

Let $M_{i}$ have output edge alphabet $\Delta_{i}$, for $i \in\{1,2\}$, and let $\$$ be a symbol not in $\Delta=\Delta_{1} \cup \Delta_{2}$. We define deterministic MSO graph-to-string transducers $M_{i}^{\$}=M_{i} ; N$ such that $M_{i}^{\$}(g)=M_{i}(g) \$$ for all $g \in \operatorname{dom}\left(M_{i}\right)$. Here $N$ is the deterministic MSO string transducer $\left(C\right.$, true, $\left.\left\{\psi_{1, \#}(x), \psi_{2, \#}(x)\right\},\left\{\chi_{c, c^{\prime}, \delta}(x, y)\right\}_{c, c^{\prime} \in C, \delta \in \Delta \cup\{\$\}}\right)$ such that $C=\{1,2\}, \psi_{1, \#}(x) \equiv$ true, $\psi_{2, \#}(x) \equiv \chi_{1,2, \$}(x, y) \equiv \neg(\exists z) \bigvee_{\delta \in \Delta} \operatorname{edg}_{\delta}(x, z)$ and, for $\delta \in \Delta, \chi_{1,1, \delta}(x, y) \equiv \operatorname{edg}_{\delta}(x, y)$; all other edge formulas are set to false.

Since now all output strings end on the special marker $\$,\left.\tau_{M_{1}}\right|_{D} \neq\left.\tau_{M_{2}}\right|_{D}$ iff

$$
\exists a \exists b:\left(d(a, b) \wedge \exists n \exists g:\left(g \in D_{1} \wedge M_{1}^{\$}(g) / n=a \wedge M_{2}^{\$}(g) / n=b\right)\right)
$$

where $d(a, b)$ denotes the statement $a, b \in(\Delta \cup\{\$\}) \wedge a \neq b$. For given $a, b$, let $M^{a, b}$ be the transducer of Lemma 6 for $a, b, M_{1}^{\$}, M_{2}^{\$}$. Then the statement displayed above holds if and only if

$$
\begin{aligned}
\exists a \exists b:(d(a, b) & \left.\left.\wedge \exists n: \operatorname{dgr}\left(a^{n} b^{n}\right) \in M^{a, b}(D)\right)\right) \\
\text { iff } \exists a \exists b:(d(a, b) & \wedge \underbrace{\left.\exists n:(n, n) \in \operatorname{Par}\left(M^{a, b}(D)\right)\right)}_{P(a, b)})
\end{aligned}
$$


By Lemma 2, $\operatorname{Par}\left(M^{a, b}(D)\right)$ is effectively semilinear. By Lemma 3 this means that $P(a, b)$ is decidable. Since there are only finitely many $a, b$ with $d(a, b)$, the statement is decidable.

We now reduce the graph-to-tree case to the graph-to-string case. Let $\Delta$ be a ranked alphabet and let $m$ be the maximal rank of its elements. There is a deterministic MSO tree-to-string transducer $M_{\Delta}$ that translates every tree $t$ over $\Delta$ into the string pre $(t)$ of its node labels in pre-order. Clearly, if we associate with a deterministic MSO graph-totree transducer $M$ (from $(\Sigma, \Gamma)$ to $(\Delta,\{1, \ldots, m\})$ ) the deterministic MSO graph-tostring transducer $\widehat{M}=M ; M_{\Delta}$, then $M_{1}$ is equivalent to $M_{2}$ on $D$ if and only if $\widehat{M}_{1}$ is


with $\psi_{1, \#} \equiv$ true, $\psi_{2, \#} \equiv \operatorname{root}(x)$, where $\operatorname{root}(x)$ expresses that $x$ is the root node. Further, for $\delta \in \Delta, \chi_{1,1, \delta} \equiv \operatorname{lab}_{\delta}(x) \wedge \pi(x, y)$ and $\chi_{1,2, \delta} \equiv \operatorname{lab}_{\delta}(x) \wedge \operatorname{root}(y) \wedge$ $\neg(\exists z) \pi(x, z)$ where $\pi(x, y)$ expresses that $y$ is the successor of $x$ in the pre-order.

String and Tree Transductions Clearly, Theorem 7 also holds if we restrict the input graphs to strings or trees. In particular, deterministic MSO $X$-to- $Y$ transducers have decidable equivalence for all $X, Y \in\{$ string, tree $\}$. For string transducers this reproves the decidability result of [Gur82] (through [EH01]). For trees we obtain the following new decidability result.

Corollary 8. The equivalence problem is decidable for deterministic MSO tree transducers.

Of course, even stronger statements hold; namely, given an NR set $D$ of strings or trees, it is decidable if two deterministic MSO $X$-to- $Y$ transducers are equivalent when restricted to $D$. For string transducers this means the following.

Corollary 9. It is decidable whether two deterministic two-way finite state transducers are equivalent on an NR set of strings.

As discussed in Section 6 of [Eng97], the NR sets of strings are the same as the ranges of deterministic tree-walking tree-to-string transducers. They properly contain, for instance, the context-free languages and the ranges of deterministic two-way finite state transducers. Since the NR sets of strings form a full AFL of Parikh languages, Corollary 9 is in fact a special case of the general decidability result for deterministic two-way finite state transducers in Theorem 5 of [Iba82]. It is incomparable to the decidability of equivalence of two such transducers on an NPDT0L language [CK87].

The two statements of the next corollary follow from the characterizations of deterministic MSO definable tree transductions in [BE00] and [EM03b], respectively. Note that a tree transducer is of linear size increase if the size of the output tree is at most linear in the size of the input tree.

Corollary 10. The equivalence problem is decidable

(1) for single-use restricted attributed tree transducers and

(2) for deterministic macro tree transducers of linear size increase. 
This result is incomparable with the decidability of the equivalence problem for nonnested separated attributed/macro tree transducers proved in [CF82]. It remains open whether the equivalence problem is decidable for attributed tree transducers and for deterministic macro tree transducers.

In [MSV03] the $k$-pebble tree transducer was introduced, and claimed to subsume (the tree translation core of) all known XML query languages. Hence, we call deterministic pebble tree transducers deterministic XML queries. Such queries can be simulated by compositions of macro tree transducers [EM03a]. If such compositions are of linear size increase, then they are MSO definable [Man03].

Corollary 11. The equivalence problem is decidable for deterministic XML queries of linear size increase.

\section{References}

[BE00] R. Bloem and J. Engelfriet. A comparison of tree transductions defined by monadic second order logic and by attribute grammars. J. Comp. Syst. Sci., 61:1-50, 2000.

[CF82] B. Courcelle and P. Franchi-Zannettacci. On the equivalence problem for attribute systems. Inform. and Control, 52:275-305, 1982.

[CK87] K. Culik II and J. Karhumäki. The equivalence problem for single-valued two-way transducers (on NPDTOL languages) is decidable. SIAM J. Comput., 16:221-230, 1987.

[Cou94] B. Courcelle. Monadic second-order definable graph transductions: a survey. Theoret. Comput. Sci., 126:53-75, 1994.

[Cou97] B. Courcelle. The expression of graph properties and graph transformations in monadic second-order logic. In Handbook of graph grammars and computing by graph transformation, Volume 1, pages 95-162. World Scientific, 1997.

[EH01] J. Engelfriet and H. J. Hoogeboom. MSO definable string transductions and two-way finite-state transducers. ACM Transactions on Computational Logic, 2:216-254, 2001.

[EM99] J. Engelfriet and S. Maneth. Macro tree transducers, attribute grammars, and MSO definable tree translations. Inform. and Comput., 154:34-91, 1999.

[EM03a] J. Engelfriet and S. Maneth. A comparison of pebble tree transducers with macro tree transducers. Acta Informatica, 39:613-698, 2003.

[EM03b] J. Engelfriet and S. Maneth. Macro tree translations of linear size increase are MSO definable. SIAM J. Comput., 32:950-1006, 2003.

[Eng97] J. Engelfriet. Context-free graph grammars. In G. Rozenberg and A. Salomaa, editors, Handbook of Formal Languages, Volume 3, chapter 3. Springer-Verlag, 1997.

[Gri68] T. V. Griffiths. The unsolvability of the equivalence problem for $\Lambda$-free nondeterministic generalized machines. J. ACM, 15:409-413, 1968.

[GS64] S. Ginsburg and E. H. Spanier. Bounded Algol-like languages. Trans. Amer. Math. Soc., 113:333-368, 1964.

[Gur82] E. M. Gurari. The equivalence problem for deterministic two-way sequential transducers is decidable. SIAM J. Comput., 11(3):448-452, 1982.

[Iba82] O. H. Ibarra. 2DST mappings on languages and related problems. Theoret. Comput. Sci., 19:219-227, 1982.

[Man03] S. Maneth. The macro tree transducer hierarchy collapses for functions of linear size increase. In Proc. FSTTCS 2003, pages 326-337, 2003.

[MSV03] T. Milo, D. Suciu, and V. Vianu. Typechecking for XML transformers. J. Comp. Syst. Sci., 66:66-97, 2003. 